\title{
Thermal evolution of an early magma ocean in interaction with the atmosphere: conditions for the condensation of a water ocean
}

\author{
T. Lebrun ${ }^{1}$, H. Massol ${ }^{1}$, E. Chassefière ${ }^{1}$, A. Davaille ${ }^{2}$, E. Marcq ${ }^{3}$, P. Sarda ${ }^{1}$, F. Leblanc ${ }^{3}$ and \\ G. Brandeis ${ }^{4}$ \\ ${ }^{1}$ IDES/CNRS/Univ. Paris-Sud, France \\ ${ }^{2}$ FAST/CNRS Univ. Paris-Sud, France \\ ${ }^{3}$ LATMOS/CNRS/Univ. of Versailles St-Quentin, France \\ ${ }^{4}$ IPGP/Sorbonne Paris Cité/CNRS, France
}

\begin{abstract}
The thermal evolution of magma oceans produced by collision with giant impactors late in accretion is expected to depend on the composition and structure of the atmosphere through the greenhouse effect of $\mathrm{CO}_{2}$ and $\mathrm{H}_{2} \mathrm{O}$ released from the magma during its crystallization. We developed a 1D parameterized convection model of a magma ocean coupled with a $1 \mathrm{D}$ radiative convective model of the atmosphere. We conducted a parametric study and described the influences of some important parameters such as the Sun-planet distance. Our results suggest that a steam atmosphere delays the end of the magma ocean phase by typically 1 Myr. Water vapor condenses to an ocean after 0.1 Myr, 1.5 Myr and 10 Myr for, respectively, Mars, Earth and Venus. This time would be virtually infinite for an Earth-sized planet located at less than $0.66 \mathrm{AU}$ from the Sun. So there are conditions such as no water ocean is formed on Venus. Moreover, for Mars and Earth, water ocean formation time scales are shorter than typical time gaps between major impacts. This implies that successive water oceans may have developed during accretion, making easier the loss of their atmospheres by impact erosion.
\end{abstract}

\section{Objectives and results}

One of the important objectives of the present work is to better understand the conditions and timing of the formation of the terrestrial ocean of water, and why such an ocean did not form on Venus, or possibly formed at an early stage but later evaporated due to the increasing luminosity of the young Sun. The presence of an early massive atmosphere of water vapor on Venus, which further escaped to space, and/or was trapped in the interior under the form of hydrates, is generally considered to have initiated the strong greenhouse effect observed today [1,2]. This massive $\mathrm{H}_{2} \mathrm{O}$ atmosphere may have resulted, (i) either from the evaporation of a primordial water ocean, followed by hydrodynamic escape of hydrogen [3], or (ii) from the outgassing of the primordial magma ocean, not necessarily followed by the formation of a (transient) water ocean [4]. Determining, through a comparative modeling approach applied to the three terrestrial planets Mars, Earth and Venus if a water ocean ever condensed on Venus and, if so, how long this ocean survived, is one of the major challenges of 
modern exobiology. So, in the present model we solve simultaneously (i) the thermal evolution of the convective magma ocean (based on Solomatov parametric scaling [5,6] and the radiativeconvective atmosphere and (ii) the volatile exchange between the magma ocean and the atmosphere. In the latter, the effects of (1) the convection in the troposphere, (2) the presence of water clouds, (3) the condensation of water vapor as well as (4) the pressure contribution of $\mathrm{N}_{2}$ gas is included. At each time, the atmosphere should be in volatile equilibrium with the magma ocean. The dynamics of the magma ocean have been simplified compared to some previous models. We do not take into account the effect of tidal heating [7] on the magma ocean cooling, nor the effect of the rotation on the convective patterns. We also do not resolve in details the sequence of petrological phases that crystallize as the magma ocean cools down [8], but we include the influence of the depthdependence of the solidus-liquidus curves on the magma ocean solidification. Moreover, we take into account the variations of the magma viscosity with temperature and crystal content in the convective modeling. We look, for instance, at different planet sizes at different distances from the sun (Figure 1). Below $400 \mathrm{Wm}^{-2}$, condensation occurs regardless of the size of the planet. Above this value, for a given magma ocean depth $(1500 \mathrm{~km}$ here $)$ and a given volatile content the smallest planets might never be able to sustain a water ocean. This is due to a lower value of atmospheric pressure. More detailed discussion can be found in $[9,10]$.

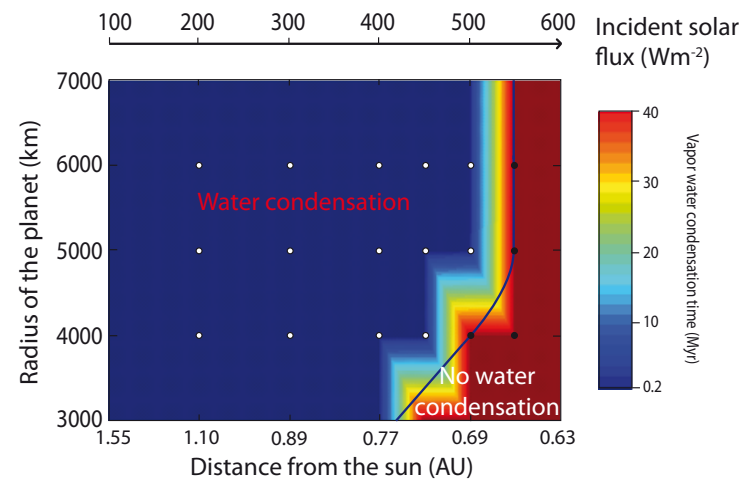

Fig. 1. Diagram showing condition for condensation of water vapor as a function of radius of the planet and solar flux. Color scale shows times at which condensation occurs. Time increases from blue to red.

\section{CONCLUSION}

This work has potentially important implications. First, it shows that Venus is close to the distance from the Sun (0.66 AU), below which, an Earth size planet remains in the magma ocean stage for a virtually infinite time. The typical time for an ocean of water on Venus to appear is of the order of 10 Myr, comparable to the duration of the main accretion sequence, suggesting that Venus could have remained in the magma ocean stage during most of its accretion. Our model shows that a water ocean may or may not have condensed on Venus after accretion, depending on the details of both IR radiative transfer and volatile inventory. A more realistic model including accretional heating due to small impacts and hydrodynamic escape is required for a more detailed analysis. Accretional heating and hydrodynamic escape of $\mathrm{H}_{2} \mathrm{O}$ are expected to play against the condensation of water, whereas hydrodynamic escape of $\mathrm{CO}_{2}$ should favors the formation of an ocean. Second, the time for condensing a water ocean on Earth and Mars is smaller than 1 Myr (1 Myr for Earth, 0.1 Myr for Mars), suggesting that a water ocean could have formed in the periods between major impacts (able to melt a significant fraction of the mantle). If serial water oceans developed on Earth and Mars 
during their accretion, impact erosion of the atmosphere could have been more efficient on these planets, explaining their relatively tenuous atmosphere with respect to Venus.

\section{References}

1. Y. Shimazu, and T. Urabe, Icarus, 9, 1968.

2. S. I. Rasool, and C. de Bergh, Nature, 226, 1970.

3. J. F. Kasting, Icarus, 74, 1988.

4. C; Gillmann, E. Chassefière, and P. Lognonné, Earth Planet. Sci. Lett. 286, 2009.

5. V. S. Solomatov and L.N. Moresi, Geophys. Res. Lett., 24, 1997.

6. V. Solomatov, Origin of the Earth and Moon, pp.323-338, 2000.

7. K. Zahnle, N. Arndt, C. Cockell, A. Halliday, E. Nisbet, F. Selsis, and N. H. Sleep,Space Science Reviews, 129, 2007.

8. L. Elkins-Tanton, Earth and Planetary Science Letters, 271, 2008.

9. Marcq, E, J. Geophys. Res, 117, 2012.

10. T. Lebrun, H. Massol, E. Chassefière, A. Davaille, E. Marcq, P. sarda, F. Leblanc and G. Brandeis, J. Geophys. Res, accepted. 
